\title{
Performance Evaluation of HTTP and DB Traffic Application Using Proactive and Reactive Routing Protocols
}

\author{
Swati Puri ${ }^{1}$, Vishal Arora ${ }^{2}$ \\ ${ }^{1}$ (CSE, S.B.S.S.T.C/PTU Jalandhar, India) \\ ${ }^{2}$ (CSE, S.B.S.S.T.C/PTU Jalandhar, India)
}

\begin{abstract}
A group of fixed or mobile communication devices that does not have a stable infrastructure to communicate with each other is known as mobile ad-hoc network. In MANET their is very importent role of nodes. All nodes can communicate with each other by directly or indirectly method. Routing is a most important part in MANET. There are three types of protocols we use for routing i.e. Proactive, Reactive and Hybrid. In this Paper, we study the AODV (Ad-hoc On-Demand Distance Vector), DSR (Dynamic Source Routing), OLSR (Optimized Link State Routing Protocol) routing protocols for DB (database) and HTTP (hyper text transfer protocol) traffic types. 20 and 45 nodes are used to computed various parameters like throughput, number of hops and response time and compare using OPNET (Optimized Network Engineering Tool) simulator.
\end{abstract}

Keywords: Mobile Ad-hoc Network (MANET), AODV, DSR, OLSR, HTTP (Hyper Text Transfer Protocol) Database (DB), Optimized Network Engineering Tool (OPNET)

\section{Introduction}

Mobile ad hoc networks (MANET) are wireless networks without fixed infrastructure. Nodes can communicate by directly or indirectly method [1]. Nodes can act as a host or a router in each time. In MANET the topology can be dynamic due to the mobility of mobile nodes. [2]. Routing protocols are mainly divided into two classes: table-driven routing protocols and on-demand routing protocols [3]. The network topology include the time and collect routes from each node to every extra node by before they are required, thus they are also called table-driven protocols [4]. In reactive protocols, routes are created when they are required by the source using a route detection method [5]. MANET is suitable for emerging situations like natural disasters, military conflicts and emergency medical situations because they involve negligible configuration and rapid deployment [6]. The main key parameter used for communication in MANET is quality of services (QoS). The most excellent paths from source to destination in calculated by QoS. [7]. QoS is in terms of bandwidth, delay or loss probability etc [8]. In this paper MANET with reactive, proactive ad-hoc routing protocols are studied and evaluated using OPNET Modeler. The performance parameters of Ad hoc routing Protocol (AODV, DSR AND OLSR) like throughput, number of hops and response time in MANET using database and http traffic application has been compared. In Section 2, we discuss MANETs routing protocols. Section 3 briefly describes the Simulation Set up. Results and Discussions have been explained in section 4. The last section presents the conclusion.

\section{Routing Protocols}

Table-driven routing protocols and on-demand driven routing protocols are the two main protocols. The routing protocols that are considered in the analysis are Ad-Hoc On-demand Distance Vector (AODV), DSR (Dynamic Source Routing) and Optimized Link State Routing (OLSR) etc.

2.1 AODV (Ad-hoc on-demand and distance vector): AODV protocol is a mixture of on-demand and distance vector. A node required to recognize a route for particular destination it creates a ROUTE REQUEST. After the request reaches a node with route to destination it creates again a REPLY. This route produced from every node as of source to destination is a hop-by-hop state [9].

2.2 DSR (Dynamic Source Routing): In Dynamic Source Routing, first node generates Route Request (RREQ), which is sent over data packet. RREQ packet is expected by every node and it is not familiar about the route to the destination. Route Error (RERR) data packet is produced and retransmitted to the route while all node cannot transmit the data packet to other nodes in the MANET [10].

2.3 OLSR (Optimized Link State Routing): Optimized Link State Routing (OLSR) is a topology based, neighbor selection protocol, where every node only maintains a subset of network topology in sequence. OLSR is a proactive protocol, for the reason that it exchanges the topology information through other nodes repeatedly to maintain information required for routing [11]. Two ways are used for optimization, firstly by reducing the 
volume of the control packets and secondly by reducing the number of links link. Each node maintains the topology information regarding the network by exchanging link-state messages between the other nodes [12]

\section{Simulation Setup}

The performance evaluation was carried out using OPNET simulator in area of $5 \mathrm{~km} \times 5 \mathrm{~km}$ using a random way point mobility model. Mobility models were created for the simulations using 20 and 45 nodes with vector mobility. The heavy browsing HTTP and medium load Database network traffic is used during simulation interval of 1200s. Mobility model used is random waypoint model with mobility of $500 \mathrm{~m}$. The implementation of the MANET network is evaluated by routing protocol such as ADOV, DSR and OLSR in different scenarios. The buffer size of data is set to 4194304 bits. Data rate is $11 \mathrm{Mbps}$ for each mobile workstation with extended rate physical 802.11g. Randomly flow of traffic is given by different HTTP and DB applications workstations that are placed at different distances as shown in Fig. 1.

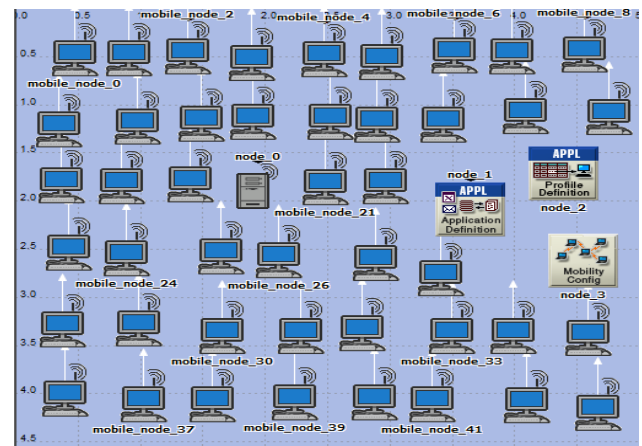

Figure 1 MANET Network Scenario for 20 Nodes

Table 1 Various Simulation Parameters

\begin{tabular}{cc}
\hline Routing Protocols & AODV, DSR, OLSR \\
\hline MAC Layer & $802.11 \mathrm{~g}$ \\
Mobile Nodes & 20,45 \\
Buffer Size & 4194304 Bits \\
MANET Size & $5 \mathrm{Km} \times 5 \mathrm{Km}$ \\
Mobility Model & Random Waypoint Mobility \\
Simulation Time & 20 Min \\
Data Rate & 11 mbps \\
Traffic Type & HTTP (Heavy Browsing) and DB \\
\end{tabular}

\section{Result And Discussion}

In this paper, we consider following four performance matrices to evaluate the AODV, DSR, OLSR routing protocols.

4.1 Throughput: The quantity of data transferred from one place to another or processed in a particular amount of time is known as throughput. Data transfer rates and networks are calculated in terms of throughput. Throughputs are considered in kbps, mbps and gbps. For 20 nodes at 10min the throughput of OLSR protocol is $165,024.50$ and at $20 \mathrm{~min}$ throughput is $157,962.91$ for HTTP Traffic. At $10 \mathrm{~min}$ the throughput is $114,376.55$ and at 20min throughput is 106,885.36 for DB Traffic as shown in Fig 2. For 45 nodes at 10min the throughput of OLSR protocol is 1,132,222.70 and at 20min throughput is 1,329,062.94 for HTTP Traffic. At 10min the throughput is 1, 114,855.38 and at 20min throughput is 1,249,668 for DB Traffic as shown in Fig 3. 

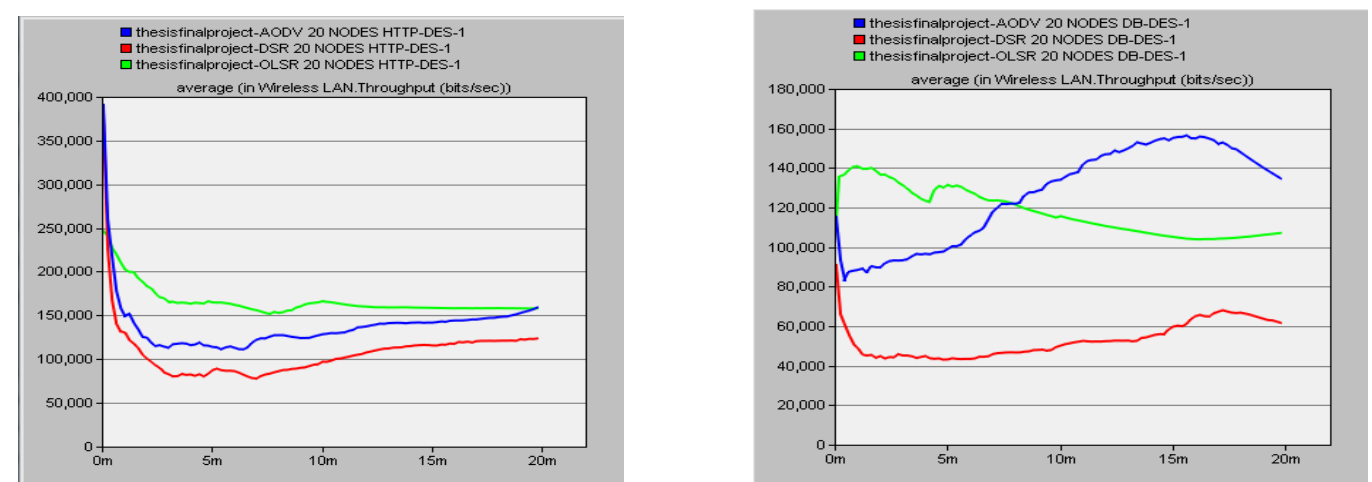

Figure 2. Throughput for 20 Nodes using HTTP Traffic and DB Traffic
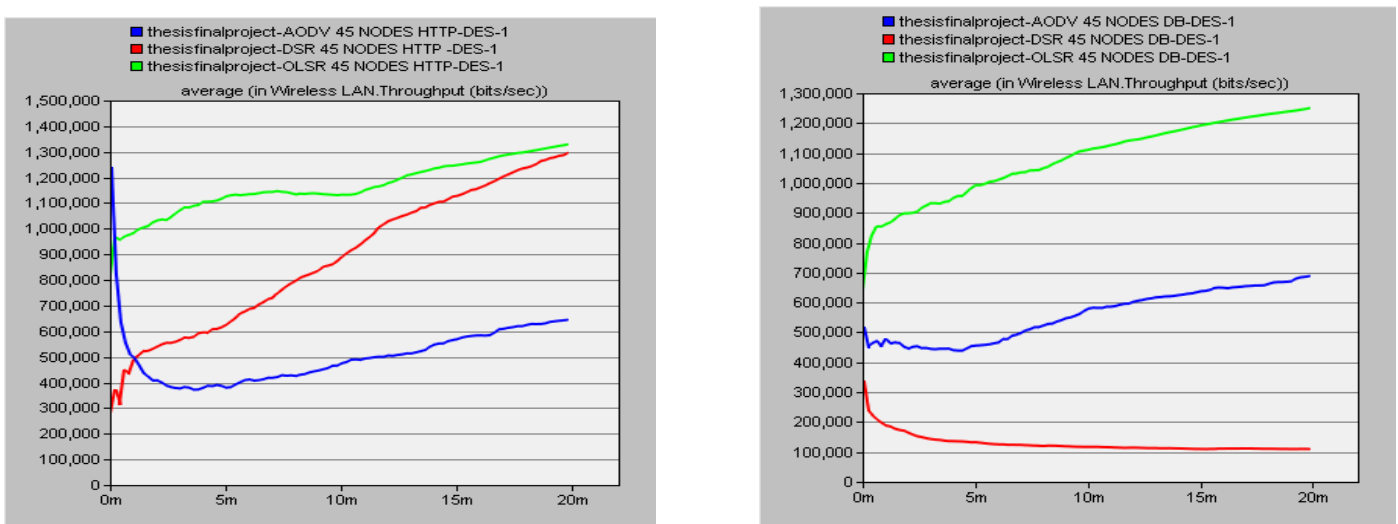

Figure 3. Throughput for 45 Nodes using HTTP Traffic and DB Traffic

4.2 Number of Hops: The statistics gives the average number of IP hops taken by data packets reaching at destination nods. For 20 nodes at $10 \mathrm{~min}$ the number of hops of OLSR protocol is 2.41246 and at $20 \mathrm{~min}$ number of hops is 2.151698 for HTTP Traffic. At $10 \mathrm{~min}$ the number of hops is 1.988467 and at 20 min number of hops is 1.59168 for DB Traffic as shown in Fig 4. For 45 nodes at 10min the number of hops of OLSR protocol is 2.275781 and at $20 \mathrm{~min}$ number of hops is 2,567225 for HTTP Traffic. At 10min the number of hops is 2.24721 and at 20min number of is 1.96581 for DB Traffic as shown in Fig 5.
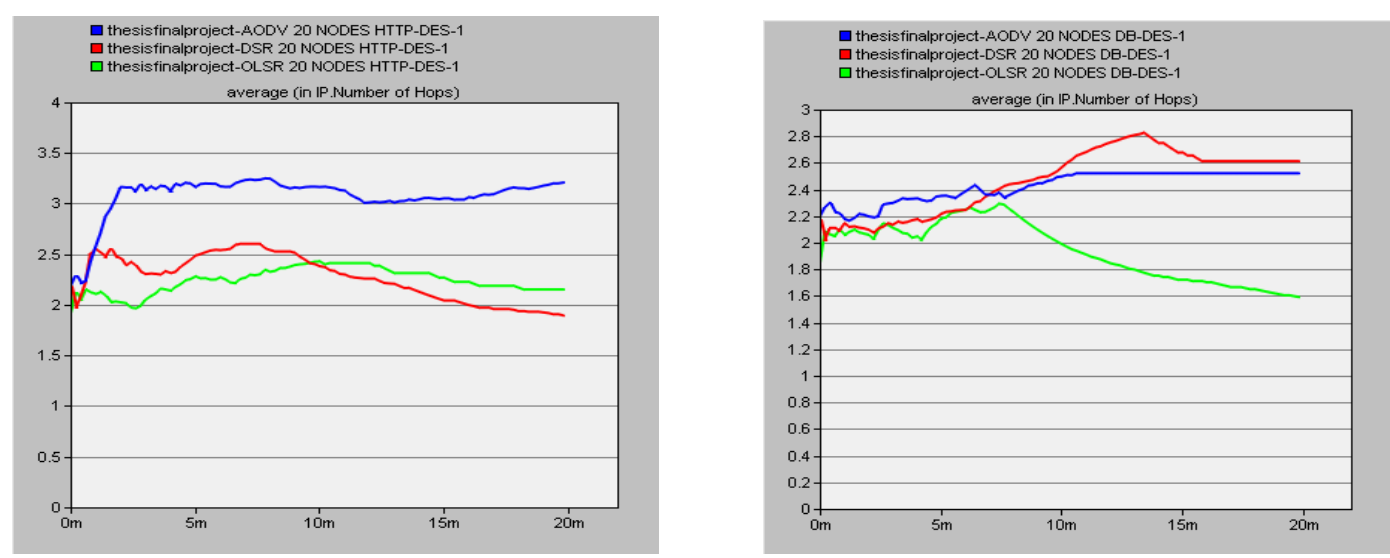

Figure 4. Number of Hops for 20 Nodes using HTTP Traffic and DB Traffic 

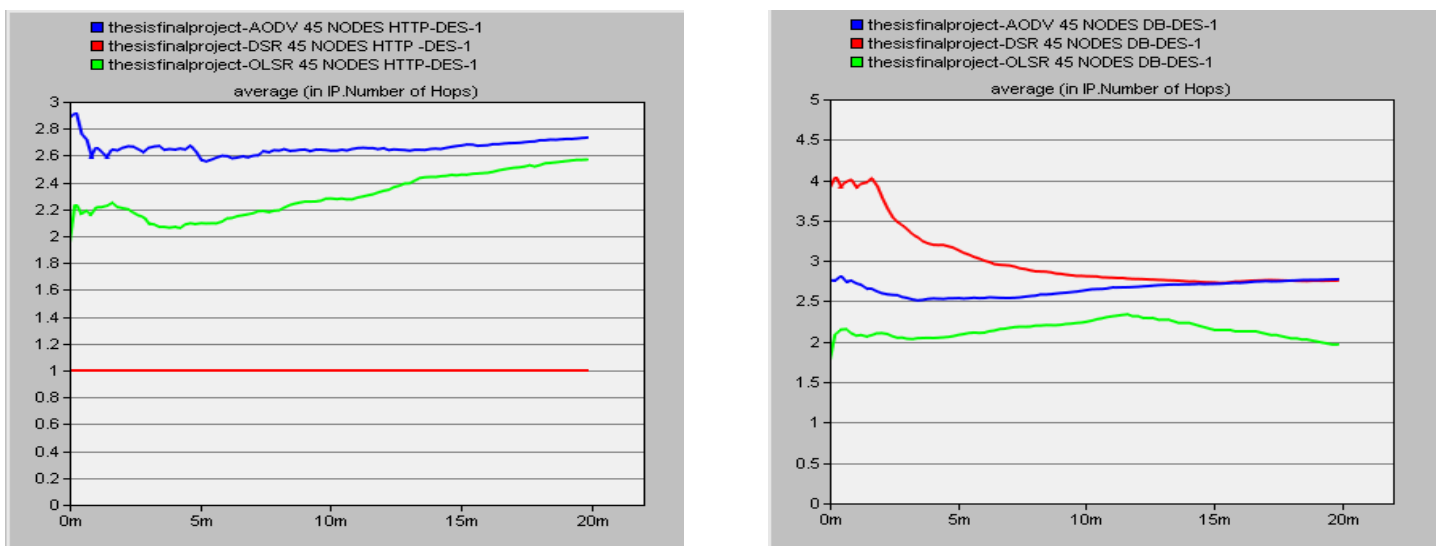

Figure 5. Number of Hops for 45 Nodes using HTTP Traffic and DB Traffic

4.3 Response Time: Time elapsed between sending a request and receiving the response from source to destination. For 20 nodes at $10 \mathrm{~min}$ the response time of OLSR protocol is 7.562528 and at $20 \mathrm{~min}$ response time is6.618174 for HTTP Traffic. At 10min the response time is 11.462434 and at $20 \mathrm{~min}$ response time is 6.729236 for DB Traffic as shown in Fig 6. For 45 nodes at 10min the response time of OLSR protocol is 3,388134 and at $20 \mathrm{~min}$ response time is $3.626768 \mathrm{HTTP}$ Traffic. At $10 \mathrm{~min}$ the response time is 6.754614 and at $20 \mathrm{~min}$ response time is 4.878302 for DB Traffic as shown in Fig 7.
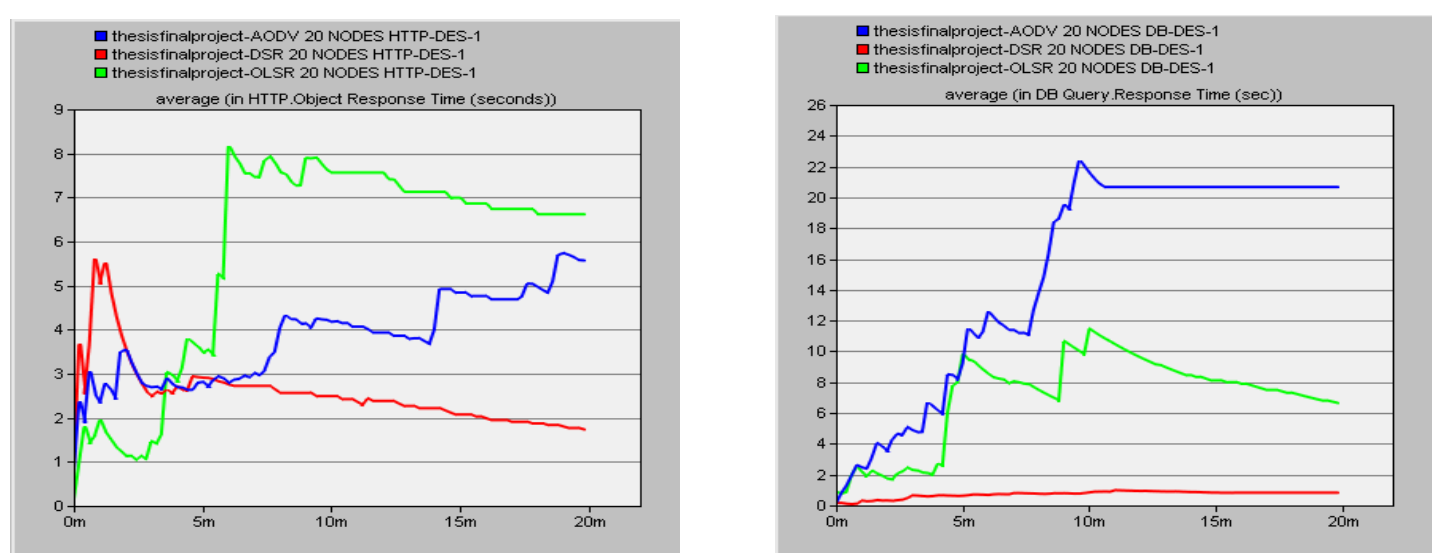

Figure 6. Response Time for 20 Nodes using HTTP Traffic and DB Traffic
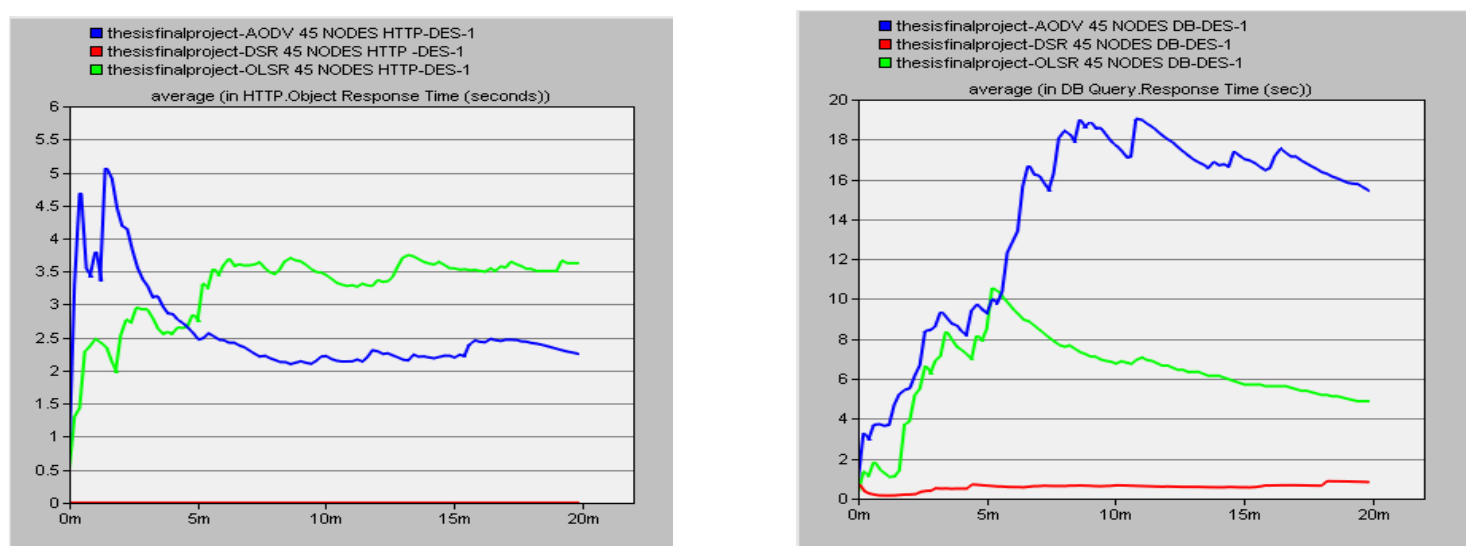

Figure 7. Response Time for 45 Nodes using HTTP Traffic and DB Traffic

\section{Conclusion}

The simulation representation of Mobile ad-hoc network is presented in this paper using OPNET. We analyzed special reactive and proactive ad-hoc routing protocols through different mobile nodes using HTTP and DB traffic application. We concluded that the general performance of OLSR using HTTP traffic application is improved preference for 20 and 45 nodes networks. The performance of DSR and AODV will changed through 20 and 45 nodes networks. The overall simulation result also shows that OLSR routing protocol is best for 
MANET, but AODV and DSR has reduced QoS with HTTP and DB traffic application. In future we can evaluate the proactive, reactive and hybrid protocols with the special traffic application like ftp, voice, text etc. In future work we can increase the data rate to check the performance matrices.

\section{References}

[1] Kim, Dongkyun, et al. "Routing mechanisms for mobile ad hoc networks based on the energy drain rate." Mobile Computing, IEEE Transactions on 2.2 (2003): 161-173.

[2] Masoudifar, Mina. "A review and performance comparison of QoS multicast routing protocols for MANETs." Ad Hoc Networks 7.6 (2009): 1150-1155.

[3] Wang, Nen-Chung, Yung-Fa Huang, and Jhu-Chan Chen. "A stable weight-based on-demand routing protocol for mobile ad hoc networks." Information Sciences 177.24 (2007): 5522-5537.

[4] Trung, Ha Duyen, Watit Benjapolakul, and Phan Minh Duc. "Performance evaluation and comparison of different ad hoc routing protocols." Computer Communications 30.11 (2007): 2478-2496.

[5] Abolhasan, Mehran, Tadeusz Wysocki, and Eryk Dutkiewicz. "A review of routing protocols for mobile ad hoc networks." Ad hoc networks 2.1 (2004): 1-22.

[6] Costinela-Luminita, C., and C. N. Magdalena. "Methods for Securing Routing Protocols in Ad-Hoc Networks." Symbolic and Numeric Algorithms for Scientific Computing (SYNASC), 2012 14th International Symposium on. IEEE, 2012.

[7] Hanzo, Lajos. Quality of Serviice-aware Routing and Admission Control for Mobile Ad Hoc Networks. Diss. University of Surrey, 2009.

[8] Er. Rupinder kaur, Er. Manjit Thapa, Er. Bhubneshwar Sharma. " Quality of service routing for mobile ad hoc networking as latest trends used in computer and electronics engineering” International Journal for Resear ch in Science \& Advanced Technologies Issue-2, Volume-4, 166-171

[9] Mohapatra, S., and P. Kanungo. "Performance analysis of AODV, DSR, OLSR and DSDV routing protocols using NS2 Simulator." Procedia Engineering 30 (2012): 69-76.

[10] Royer, Elizabeth M., and Chai-Keong Toh. "A review of current routing protocols for ad hoc mobile wireless networks." Personal Communications, IEEE 6.2 (1999): 46-55.

[11] Kuosmanen, Petteri. "Classification of ad hoc routing protocols." Finnish Defence Forces, Naval Academy, Finland, petteri. kuosmanen@mil.fi (2002).

[12] Gupta, Anuj K., Harsh Sadawarti, and Anil K. Verma. "Review of various Routing Protocols for MANETs." International Journal of Information and Electronics Engineering 1, no. 3 (2011). 